\title{
Attending to scientific practices within undergraduate research experiences
}

\author{
Gina M. Quan ${ }^{1}$, Chandra Turpen ${ }^{1}$, and Andrew Elby ${ }^{1,2}$ \\ ${ }^{I}$ Department of Physics, University of Maryland, College Park, 082 Regents Drive, College Park, Maryland, 20742 \\ ${ }^{2}$ Department of Teaching and Learning, Policy and Leadership, University of Maryland, College Park, 2311 Benjamin \\ Building, College Park, Maryland, 20742
}

Ford (2015) argues for viewing "scientific practice" not as a list of particular skills, but rather, more holistically as "sets of regularities of behaviors and social interactions" among scientists. This conceptualization of scientific practices foregrounds how they meaningfully connect to one another and are purposefully employed in order to explain nature. We apply this framework in the context of undergraduate research experiences (UREs) to understand the early forms of student engagement in scientific practices, and how these specific forms of engagement may be consequential for students' future participation. Using video from interviews with students and research mentors, we argue that this "practice" lens affords new insights into understanding students' experience of UREs. We also use this data to illustrate how coming to engage in scientific practices might look in early stages of participation.

\section{INTRODUCTION}

Prior research on undergraduate research experiences (UREs) suggests that UREs can have a variety of benefits, including the development of content knowledge, the acquisition of skills, and the building of meaningful relationships. But across a variety of methodological approaches, including surveys [1-2] and ethnographic studies [3-4], most studies on UREs have focused on whether students develop individual skills (e.g. lab techniques, reading, presenting, etc.), engage in particular tasks, or develop specific productive beliefs. These studies typically characterize experiences as more positive when students engage in more of these isolated outcomes [1-4].

This work has identified many positive outcomes of UREs. However, we argue for a more holistic perspective, which takes into account the interactions of these outcomes, for two reasons: 1) Our intuitive sense that doing science is more than just the enactment of certain kinds of knowledge and skills; it also relies on seeing how each of these are meaningfully connected and embedded within a broader purpose, and 2) Our commitment to viewing learning science as a process of legitimate peripheral participation [5], in which learning and doing science are not reducible to the accumulation of specific skills and knowledge. We selected Ford's framework [8] to model legitimate peripheral participation in physics UREs because it draws attention to the holistic features of scientific activities.

As other PER scholars have described, coming to do science can be conceptualized as a process of becoming a more central participant within a community of practice [67]. Legitimate peripheral participation (LPP) refers to the process of novices becoming more central by engaging in joint work with experts [5]. This perspective studies how one's participation shifts through interactions with others.

To study LPP in physics research, we ask, what does it look like for newcomers in physics research to engage peripherally in authentic scientific practice? In this paper, we show how Ford's practice framework can be useful for characterizing early examples of LPP in authentic science.

\section{SCIENTIFIC PRACTICES}

Ford's holistic view of scientific practices builds from the Next Generation Science Standards (NGSS). While the NGSS outlines several practices for K-12 (e.g., modeling, formulating questions), Ford foregrounds that its purpose is not to enumerate individual practices but rather to draw attention to how they function in relation to one another and to the broader scientific enterprise [9].

Ford describes three aspects of practices [8] (for brevity, we omit the third aspect):

1) Connectedness- Practices make sense in terms of one another.

2) Purposefulness- Practices make sense in terms of their ability to explain nature.

For both of these aspects of practice, appropriate participation also anticipates and responds to critiques of the practices' connectedness and purposefulness.

This framework provides a language to describe the extent to which students' participation in science is more central or peripheral, where central participation involves being able to understand and articulate the connectedness and purposefulness of scientific practices. Moreover, Ford discusses these features of practices as idealized end-goals for scientific engagement, and challenges researchers and practitioners to think about how one scaffolds early engagement in practices. Applying this framework in early UREs can also shed light on how some forms of peripheral participation in scientific practices may impact ways that students participate in research in the future.

\section{CONTEXT AND METHODS}

This study is embedded within a larger multi-year study of first-year physics majors' UREs. Students in the study enrolled in a course at the University of Maryland, which 
has two components: 1) Working in pairs with graduate student and faculty mentors on research projects outside of class and 2) participating in a weekly seminar, with a separate instructor, where they developed research skills and reflected on their experiences. In one semester, we invited all 17 students to participate in pre- and postinterviews. For the twelve students we interviewed, we invited all six of their mentors to participate in interviews. Interview questions asked students and mentors to describe their research projects, and probed students' attitudes toward research.

We selected two focal groups, each group containing 1 mentor and 2 students, for our preliminary analyses based on having interviews from both students and the mentor. We began by viewing and transcribing the interviews and flagged moments in which students or mentors described something aligned or misaligned with one of Ford's features of practice. In our analysis, we identified practices as they emerged in student interviews, rather than looking for ones specifically described in the NGSS. We then developed analytic memos in which we used transcript segments to develop claims about how students engaged in and how mentors supported connectedness and purposefulness [10]. We refined our analyses by synthesizing our accounts of members of the same mentormentee research teams [11]. These analyses and video data were also presented at research group meetings to identify the claims that were best supported by the data [10].

In this paper, we present preliminary analyses of two research trios, each with one mentor and two undergraduate students. We use the first analysis to suggest the utility of the practice lens for studying research experiences, and the second analysis to describe what some of the processes of LPP might look like within a research setting.

\section{JACK, FRANK, AND ARTHUR: UTILITY OF PRACTICE LENS}

Jack mentored Frank and Arthur on a computational physics project modeling plasma in the ionosphere. Frank had transferred from another university and was taking advanced physics courses, and Arthur was a transfer student taking intro level courses. In Jack's interview, he emphasized the importance of connectedness and purposefulness, and contrasted that with other kinds of research experiences where you just "turn the crank" and don't understand what you're doing. We found evidence that the students also experienced aspects of connectedness and purposefulness with respect to scientific practices.

\section{A. Evidence of connected practices}

When asked to describe a "typical day in research," Frank drew connections between computational and theoretical aspects of his research, as well as how their engagement connected to scientific critique.
Jack would explain to us what the objective was for the day. Whether it was basic coding towards the beginning of the sessions or theory of the plasma frequency and the index of refraction. He lays down the groundwork, and then we go in. We start coding exactly what we think should happen... from what we know, and then submit that to Jack, he would look it over, and then we confer... Or it would be Jack gives us a code and tells us to play around with it and see what we can do... Arthur and I then figure out whether our ideas are aligned, whether they're not aligned, what makes sense, what doesn't make sense. And so it would be a group project, where we go back and forth. We all have a third of the project to do. And, we confer and we make it a whole.

Within this description, there is evidence that practices are connected for Frank. He outlines several activities such as "laying down the groundwork," enacting computation, and engaging in evaluation of those performances. Frank's use of the transitions "and then" between the practices suggests that they are connected sequentially. The coding processes also directly connect to their conceptual knowledge ("we start coding exactly what we think should happen... from what we know") suggesting that the computational processes stemmed from conceptualizing predictions of what they thought would happen. In the second half of the quote, Frank describes another mode of engagement in which they explore a code that had been previously developed by other researchers. Using this exploration of previously developed code, they figure out "whether our ideas are aligned" with one another's. This critique is connected to the understanding they developed through previous exploration of the code. Frank's description of how scientific practices flow into one another makes sense to us as researchers and helps us understand how Frank is making sense of the logic behind his inquiry.

Frank's quote also suggests some of the ways in which Jack scaffolded participation in connected practices. Jack set objectives to frame the activities, and did some critique of their performances of computation. In the next section, we describe how these practices were embedded within a scientific purpose. Frank's partner, Arthur, describes similar connectedness in the next section, as well as evidence that the practices were purposeful in certain ways.

\section{B. Evidence of purposeful practices}

Arthur articulated how the research practices of his project connected to a broader scientific aim.

So, we wanted to accomplish two objectives...our first objective was to prove that the radiation that was detected in the South Pole was the same radiation that was scattered off the plasma in our ionosphere. And our second objective was to find out the exact path the ray took in coming to the South Pole. To do that we had to 
first off learn about MATLAB... But the more intensive part was developing and deriving the ray tracing equations used to calculate the path the ray took.

Arthur describes the broader physical system within his description of the research objectives, demonstrating the purposefulness of his engagement. His use of the transition "to do that we had to..." suggests that these practices were directly embedded within this scientific purpose.

Frank similarly describes the scientific purpose of their activity and how it emerged from prior research:

Well basically we built a theoretical model of what was already done by researchers in the North Pole... nobody has ever actually traced the path or given a concrete, a concrete statement saying that "oh this is definitely possible"... We just made a model of what potentially was what made it to the South Pole.

Frank situates his research in terms of prior research and what is unknown. They used representational tools (ray tracing in a waveguide) to develop a computational model which would answer a question, whether radiation in the ionosphere could scatter to the South Pole. Frank's description not only states that their research is purposeful because it answers an unknown question, but also presents a coherent account of how each of these steps connect in order to achieve a scientific purpose.

Within this paper, our purpose is to demonstrate how the practice framework gives a language for describing two aspects of scientific practice, connectedness and purposefulness. Moreover, our analysis brings attention to how these practices "hang together" and invites evaluation of the sensibility, plausibility, and coherence of the logic behind students' accounts of their experiences.

The next pairing illustrates how Ford's framework helps us characterize LPP in authentic science practices.

\section{SIMON, NEIL, AND LIAM: EXTENDING PRACTICE TO LPP}

\section{A. Research context as described by mentor}

Simon mentored Neil and Liam in a lab studying BoseEinstein Condensates (BECs). Their project was to design a device to determine if temperature fluctuations in the lab were impacting the experiment. Simon had intentionally structured the research project to support connectedness:

So the theme is make some widget, use widget to measure something we didn't know... That whole combined package of that is really what I want out of these projects for them.

Simon emphasizes the connectedness of designing a "widget," building it, and using it to conduct an investigation. Neil and Liam's interviews show evidence that they took up these practices as being connected to one another; however, there was also evidence that they did not understand the broader scientific purpose of their work.

\section{B. Peripheral participation in purposeful practices}

We now describe the peripheral nature of Liam and Neil's engagement in scientific practices with respect to the purposeful dimension. In Neil's interview, he was able to describe why the practices he engaged in were relevant to the lab's experiment, but not how the experiment itself fit into a broader scientific research purpose.

Neil: We were working in a lab that dealt with BoseEinstein Condensates... what they had saw was that there was fluctuations in the current and they weren't sure why. They thought it might have been temperature, and so me and my partner designed a circuit to measure temperature... then see if there's a correlation between the temperature and the changes in the current. And it turned out there seemed to be...

Interviewer: Alright, how much did you feel like you understood how your research fit into the broader goals of the lab?

Neil: Um, well, I'd say not very well. I mean I understood that they're trying to clean up some data and remove some weird fluctuations but why they're measuring the uh the electric fields of the Bose-Einstein Condensates, that sort of stuff I didn't really understand.

Neil experiences the practices as being purposeful, but his participation is peripheral; he describes that the widget helps the lab "clean up some data." On the other hand, the broad importance of that data is opaque to him, and he notes that. While he is able to participate peripherally in some practices of the lab by understanding how his project helps the experiment, he hasn't gained "deeper" membership by understanding the broader purpose of the experiment. Later in the interview, Neil associates developing a broader understanding of the physics purpose with taking more physics classes. This suggests that he sees physics coursework as necessary for fuller participation.

The example of Neil suggests that in this case there are at least two aspects of the "purposefulness" of a scientific practice. One is seeing how one's activity can contribute to a particular experiment. The other involves understanding the scientific "point" of the BEC experiment and why removing current fluctuations would help. Peripheral participation in purposeful practice, as illustrated by Neil, can involve believing that BECs are scientifically important and that his work is contributing to understanding them better, and perhaps that he'll have access to a deeper understanding in the future.

In Liam's interview, he similarly does not describe the broader purposes of the experiment and focuses on the 
utility of the circuit they built. Unlike Neil, however, Liam suggests that experimentalists don't need to understand the broader theory of a research project: "Theorists just give me things to work on. They say 'I have a problem here, can you test it?' Like, sure I'll design something and work on it." Liam's description suggests that only theorists get access to the broader scientific purpose of experimental work.

Liam and Neil's experience demonstrates that LPP within a research lab can involve understanding narrow aspects of the scientific purpose-how it contributes to a given experiment-but not fully understanding the broader scientific purpose. We note differences in the peripheral nature of their engagement; Liam describes it as a fixed feature of doing experimental work, whereas Neil attributes it to his currently limited background knowledge and coursework. Applying the practices framework helps us explore these differences.

\section{DISCUSSION}

We applied Ford's framework to illustrate the utility of attending to the connectedness and purposefulness of undergraduate researchers' engagement in scientific practices as a way to understand their LPP. While prior research on UREs measures engagement in different activities as independent outcomes, layering on Ford's practice framework gives a more holistic sense of students' participation in physics practices.

Methodologically, we also find value in this analysis of interviews. While surveys can capture the degree to which students view scientific practices as purposeful or connected, interviews allow us to characterize the nature of that purposefulness and connectedness. Consider the survey item "I understand the broader purpose of the experiments I am conducting." [12] Frank would likely agree with that item, but his survey response wouldn't allow us to examine how his participation in practices "hangs together" and the sensibility, plausibility, and coherence of the logic behind those practices. In a different vein, Liam and Neil might both disagree with that item, but for different reasons: Liam's response might reflect how he thinks theorists dictate experimental physics, whereas Neil might see

[1] D. Lopatto, Cell Biology Education 3, 4 (2004).

[2] R. Taraban \& E. Logue, J Educ Psychol, 104, 2 (2012).

[3] A. B. Hunter, S. L. Laursen, and E. Seymour, Sci. Ed. 91, 1 (2007).

[4] S. L. Laursen et. al., Undergraduate research in the sciences (John Wiley and Sons, San Francisco, 2010).

[5] J. Lave \& E. Wenger, Situated Learning: Legitimate Peripheral Participation (Cambridge University Press, Cambridge, 1991).

[6] P.W. Irving \& E. C. Sayre, Phys. Rev. ST-PER. 10, 1 (2014).

[7] E. W. Close, J. Conn, \& H. G. Close, Phys. Rev. STPER. 12, 1 (2016). himself as able to understand the broader purpose in the future. Analysis of interviews using Ford's framework lets us characterize these different forms of LPP.

This work also suggests features to foreground when supporting undergraduate researchers or developing courses such as the one in this study. We argue that URE mentors should attend to the ways in which scientific practices are meaningful with respect to one another and to a broader scientific purpose, and the logic behind how these aspects of practice are coordinated, from both the mentor's and the students' perspective. For example, mentors can design research experiences such that each activity informs the next. This can't all happen right from the beginning, of course, but it is important to think about how practices might come to be connected to one another and to a scientific purpose in coherent ways.

Given the preliminary nature of this study, future work will refine our classifications of connectedness and purposefulness in interview data. We will also continue to map out the different ways that students come to participate in connected, purposeful practice, and how their trajectories are impacted by mentors, subfields of physics, and structure of the URE. We will also explore the implications of the different forms of LPP in which students engage. For example, Neil suggested that he gained confidence from the fact that he didn't need to understand the broader research project. In contrast, Arthur described feeling overwhelmed by the amount of physics and math knowledge that he had to learn in a small amount of time. Together, these cases suggest that students' affect may be intertwined with their engagement in connected, purposeful practices in nuanced ways. In addition to characterizing different forms of LPP in research experiences, we plan to study how those forms impact students' future engagement in physics research.

\section{ACKNOWLEDGEMENTS}

The authors thank members of UMD PERG/Sci. Ed., Dimitri Dounas-Frazer, and Enrique Suarez for their thoughtful feedback. This work is supported by NSF DUE1245590 and the UMD Physics Department.

[8] M. J. Ford, Sci. Ed. 99, 6 (2015).

[9] H. Schweingruber, T. Keller, \& H. Quinn (Eds.). A Framework... (National Academies Press, Washington, DC, 2012).

[10] B. Jordan \& A. Henderson Journal of the Learning Sciences 4, 1 (1995).

[11] R. A. Engle, F. R. Conant, \& J. G. Greeno, in Video research in the learning sciences, edited by $\mathrm{R}$. Pea, $\mathrm{B}$. Barron \& S. J. Derry. (Routledge, NY 2007).

[12] B. G. Geller, P. Killion, W. Losert, \& C. Turpen, poster presented at the American Association of Physics Teachers 2015 Annual Meeting. 